\title{
Measurement of Learning Process by Semantic Annotation Technique on Bloom's Taxonomy Vocabulary
}

\author{
Jirawit Yanchinda ${ }^{1}$, Pitipong Yodmongkol ${ }^{1} \&$ Nopasit Chakpitak $^{2}$ \\ ${ }^{1}$ College of Arts, Media and Technology, Chiang Mai University, Chiang Mai, Thailand \\ ${ }^{2}$ International College, Chiang Mai University, Chiang Mai, Thailand \\ Correspondence: Jirawit Yanchinda, College of Arts, Media and Technology, Chiang Mai University, Chiang Mai, \\ Thailand. E-mail: jirawit.y@gmail.com
}

Received: June 21, 2015 Accepted: July 29, 2015 Online Published: December 29, 2015

doi:10.5539/ies.v9n1p107 URL: http://dx.doi.org/10.5539/ies.v9n1p107

\begin{abstract}
A lack of science and technology knowledge understanding of most rural people who had the highest education at elementary education level more than others level is unsuccessfully transferred appropriate technology knowledge for rural sustainable development. This study provides the measurement of the learning process by semantic annotation technique on Bloom's Taxonomy vocabulary to prove the effectiveness of tutorial science ontology improving the learning process of non-science and technology educated farmers. The result showed that (i) experimental sample group who additional social science ontologies significantly improve learning process of throughput domain knowledge. (ii) The experimental group reaches analysis level while the control group without ontology input meets apply level. The experimental group cognitive level improves one step further of the control group.
\end{abstract}

Keywords: innovative semantic annotation, Bloom's Taxonomy, learning process measurement, CommonKADS

\section{Introduction}

According to Thai education system provides nine years at elementary education level: Pratom 6 of compulsory education, with twelve years at lower secondary education level: Mattayom 3 of free basic education guaranteed and set by the 1999 National Education Act. Then the compulsory education was extended to nine years, with all students expected to complete with fifteen years: Mattayom 6 in 2003 (Ministry of Education, Thailand, 2008). Most of rural people in Thailand had the highest education at elementary education level that are elementary school (Pratom 4-Pratom6) at 92.58\%, lower secondary school (Muttayom 1-3) at 46.82\% and upper secondary school (Muttayom 4-6) at 25.29\%. (Office of the Permanent Secretary, Ministry of Education, 1992). Additionally, it can be seen from Office of the Permanent Secretary, Ministry of Education, (2013) that there are $91.36 \%$ of rural people who are farmers in Thailand completed elementary education level more than other levels. There are no basic science, social science and technology subjects in Thai's curriculum of the compulsory nine years of Thai education system in 1999. Additionally, the Thailand culture is agricultural community and the today age of most rural farmers is more than 35 year old who has the highest education at elementary education level in the compulsory nine years of Thai education system and need to do a farm in their own community. The findings highlight a lack of basic science, social science and technology knowledge understanding in basic education level of rural community people in Thailand being unsuccessfully transferred appropriate technology knowledge, which is maintained in the sustainable development projects. Moreover, non-science and technology educated people could not understand and apply science and technology knowledge to develop or solve problem issue of their community.

This study used tutorial science ontologies in order to prove non-science and technology educated farmers improve learning process. So, this research provides the measurement of learning process by semantic annotation technique on Bloom's Taxonomy vocabulary to prove that the additional science ontologies can improve cognitive level of non-science and technology educated farmers reflect in competence of learning process.

Learning process is a performing procedure or using various methods, which help individual learners to create their own knowledge and to design learning method how to learn (Liyabage, Strachan, Penlington, \& Casselden, 2013). The learning process includes a training method and a learning measurement, which is truly effective, the 
training method must be appropriate for the individuals and the situation. The individual or group learning development and measurement cover the obvious skills and knowledge required for the community or qualification. The individual effective development could be also considered in term of personal potential, individual learning styles and whole individual life skill development. The learning process could be approached by flexible methods of learning process design, knowledge dissemination and learning process measurement. These concepts provide for working class training, development and assessment. The method of assessing the learning process that is currently available can measure individual cognitive and skill which usually uses Bloom's Taxonomy Pappas, Pierrakos and Nagel (2013), Valcke, De Wever, Zhu, and Deed (2009), Plack, Driscoll, Marquez, Cuppernull, Maring, and Greenberg (2007).

The measurement has played principal roles in the learning process of teaching, training or coaching courses. A suitable design measurement accurately could reveal the learner's skill level of learning process. The appropriate assessment could provide learning situations of learning process for learners and validate training strategy for trainers.

This study aims to assess domain knowledge throughput of organic rice farming knowledge and to measure learning process improvement using effective tutorial science ontologies by semantic annotation techniques on Bloom's Taxonomy vocabulary. This research focuses on measurement of learning process of the working class training on organic rice farming knowledge for non-science and technology educated organic rice farmers. The learning process is designed for non-science and technology educated organic rice farmers in this research using an effective science and technology ontologies to train experimental sample group before learning organic rice farming from experts.

This research designs the measurement using innovative semantic annotation technique on Bloom's Taxonomy vocabulary to assess learning process of non-science and technology educated farmers on organic rice farming knowledge from trainers. A semantic annotation technique can connect specific keyword on Bloom's Taxonomy vocabulary to concepts and background knowledge stored in domain knowledge and ontology. The learning process outputs constitute the valuable knowledge for particular domain knowledge, however, due to the unstructured nature of the learning process output, the machines for automation purpose could not efficiently exploit this domain knowledge. Consequently, the creation of innovative semantic annotation related to Bloom's Taxonomy vocabulary seems to be a technique to exploit this domain knowledge and extract contained and obvious expertise. This study proposes semantic annotation technique on Bloom's Taxonomy vocabulary to measure the learning output and outcome on organic rice farming learning process of non-science and technology educated farmers in Phrao district, Chiang Mai province, Thailand as a case study. Most rural people in Phrao District had highest education at elementary school level at $53.4 \%$ and their ages are about more than 35 year old and they are farmers. Consequently, rural farmers at Phrao District, Chiang Mai Province, Thailand are suitable sample group for this study.

\section{Literature Review}

\subsection{Bloom's Taxonomy}

Bloom's Taxonomy has a significant influence on educational theory and practice. Bloom's taxonomy of educational aims was established as a tool for a variety of purposes. Bloom's taxonomy is systematized from simple to complex and specific to abstract cognitive classifications (Krathwohl, 2002), representing an aggregate framework that has been broadly applied in educational research (Kunen, Cohen, \& Solman, 1981). More particularly, Bloom's categories reveal levels in knowledge construction (Bloom, Engelhart, Furst, Hill, \& Krathwohl, 1956). Structuring knowledge indicates drive from basic descriptive explanations of opinion to operating a variety of cognitive strategies, such as analysis, evaluation and creativity (Anderson et al., 2001).

The cognitive process dimension on Bloom's revised taxonomy is alienated into remember, understand, apply, analyze, evaluate and create (Anderson \& Krathwohl, 2008) which represents the approach of thinking and learning process for a learner and supports the learners to maintain and transfer the learning knowledge.

The Bloom's revised taxonomy focuses to assess the learning process of learner in terms of appropriate cognitive process and knowledge form then the trainers will apply in learning process measurement to support the instructive potency, instructional assessment analysis, curriculum design etc. (Box, 2004; Chyung \& Stepich, 2003; Lister \& Leaney, 2003; Scott, 2003; Airasian \& Miranda, 2002; Mayer, 2002).

\subsection{Ontology}

The "ontology" can be expressed as an explicit specification of conceptualization. The structure of the domain can be captured via ontology, i.e. conceptualization. The conceptualization clarifies about the domain knowledge, 
not particularly about the state of affairs in the domain. Subsequently, ontology is specification of this conceptualization which using particular modelling language and particular terms specify the conceptualization. The official specification is essential in order to be able to process ontologies and automatically activate on ontologies.

The ontology defines a domain, while a knowledge base (based on ontology) particularly explains the state of relationships. A knowledge-based system has its own knowledge base, and only what can be communicated using ontology can be stored and reused in the knowledge base. When an agent desire to communicate to another agent, who uses the concepts from some ontology to understand in communication, ontologies must be shared between agents (e.g., Anumba et al., 2008; Chou et al., 2008; Saito et al., 2007; Uschold \& Gruninger, 1996).

The methodology in this research utilized ontology-based knowledge as knowledge representation to reason domain knowledge on the organic rice farming of sustainable development projects The learning process measurement in this research uses semantic annotation technique on Bloom's Taxonomy vocabulary to annotate ontologies output and domain knowledge of samples' answer test and interview questions.

\subsection{CommonKADS}

CommonKADS viewpoint for Common Knowledge Acquisition and Design System, which is a current form of KADS. The approach purposes to support structured knowledge engineering. It signifies the bottlenecks and opportunities in the organizations, allocates and applies their knowledge resources, and so provides toolkits for corporate knowledge management. It also provides the approaches to present a comprehensive analysis of knowledge-intensive tasks and procedures. CommonKADS provides the development of knowledge systems that provide designated parts of the business process (Schreiber, 1999).

Knowledge model provides the way of expert thinking, which people learn from could think as an expert. The CommonKADS model has a variety of components such as the organization model, task model, agent model, knowledge model, communication model and design model. This research focuses on the knowledge model to structure the appropriate technology knowledge from sustainable development projects based on science reasoning: biology, physics, chemistry and mathematics based framework derived from problem consideration, conceptualization, reasoning and implementation of a solution. This research focuses on CommonKADS to capture knowledge, which is one of the most widely used knowledge engineering methodologies.

\subsubsection{Annotation}

Annotation approaches can associate with a particular domain in a document to broader concepts and valuable domain knowledge stored in a knowledge base and ontology (Handschuh, 2005). The typical principle of annotation is not about trying to attribute a label to every word but it is finding those things, which are listed in the instructions. The annotations could not intersect with each other, or be contained within each other, unless the specific instructions allow this. Moreover, all set of annotations must be the work of a single annotator only and will be of an only document in separation, and would not consider other documents. Machines for automation purpose could not proficiently achieve the concepts, domain knowledge or ontology from unstructured document (Gruber, 1993). Accordingly, the creation of semantic annotation associated with document content.

\subsubsection{Semantic Annotation}

Semantic Annotation (SA) attaches a term or extent of a particular domain to a semantic database or ontology or domain concept where additional information and knowledge are stored. The semantic annotation approach proposes a framework of semantic web for constructing such metadata that it denotes to the procedure of indexing and retrieving useful domain knowledge from documents, therefore, generating annotation or metadata on top of documents. This annotation is well expressed for a specific domain using appropriate syntax and semantics, thus, the objective of semantic annotation is to create metadata, which can be exploited by both manual annotation and automated annotation by machines.

There are two basic semantic annotation tools for producing semantic annotations, which are automatic and manual semantic annotation. The automatic annotation provides recommendations for annotations that could operate many more documents. However, this automatic annotation is less precise and still requires intervention by knowledge workers. The most fundamental annotation tools allow annotators to manually generate annotations that are more accurate and in common with virtuously documented annotation tools but provide some support for ontologies. This research uses manual semantic annotation technique on Bloom's taxonomy vocabulary to measure learning process of non-science and technology educated organic rice farmers (Uren et al., 2006). 


\section{Research Methodology}

The experimental research designs on adaptive organic rice-farming samples as a learning process study samples because this group represents a research problem. The adaptive organic rice farmers were selected by taking a test that was in science and technology in organic rice farming concepts and designed by a researcher. There are ten adaptive organic rice farmers as research samples that are non-science and technology educated people who could not answer the test. Consequently, these adaptive organic rice farmers were divided equally into two groups, i.e., control group and experimental group. The only experimental group was trained social science ontologies before organic rice farming train.

This study focuses on learning process measurement of the organic rice farming knowledge by semantic annotation technique on Bloom's Taxonomy vocabulary in order to prove the effectiveness of tutorial science ontologies that can improve learning process of non-science and technology educated farmers. The social science ontologies were used as knowledge representation to transfer knowledge to rural farmers of Phrao District, Chiang Mai Province, Thailand as a research case study. The vocational learning was experimented with Bloom's Taxonomy framework to evaluate additional ontologies and vocational lifelong learning of case study.

\section{The research methodology and findings verification and validation}

The study findings were verified by experts who expertise in appropriate technology knowledge in each step this research. All tests (Q1-Q5) of this study were designed and verified by experts via teaching back verified all captured domain knowledge. The research findings were validated using test (Q1), pre-test (Q2), post-test (Q3) that were design by experts and knowledge validation (Q4, Q5) shown in Figure 1. The test Q1, Q2 and Q3 should be the same set of questions which based on Bloom's Taxonomy vocabulary. Moreover, the test Q4 and Q5 should be the same set of questions which based on Bloom's Taxonomy vocabulary as well. Experts who are the expert in appropriate technology knowledge and researcher who has science background designed and verified the tests and interview question guideline for knowledge validation in Q1, Q2, Q3, Q4 and Q5. The research experimental process was validated with the test, pre-test, post-test and knowledge validation. (Shown in Figure 1)

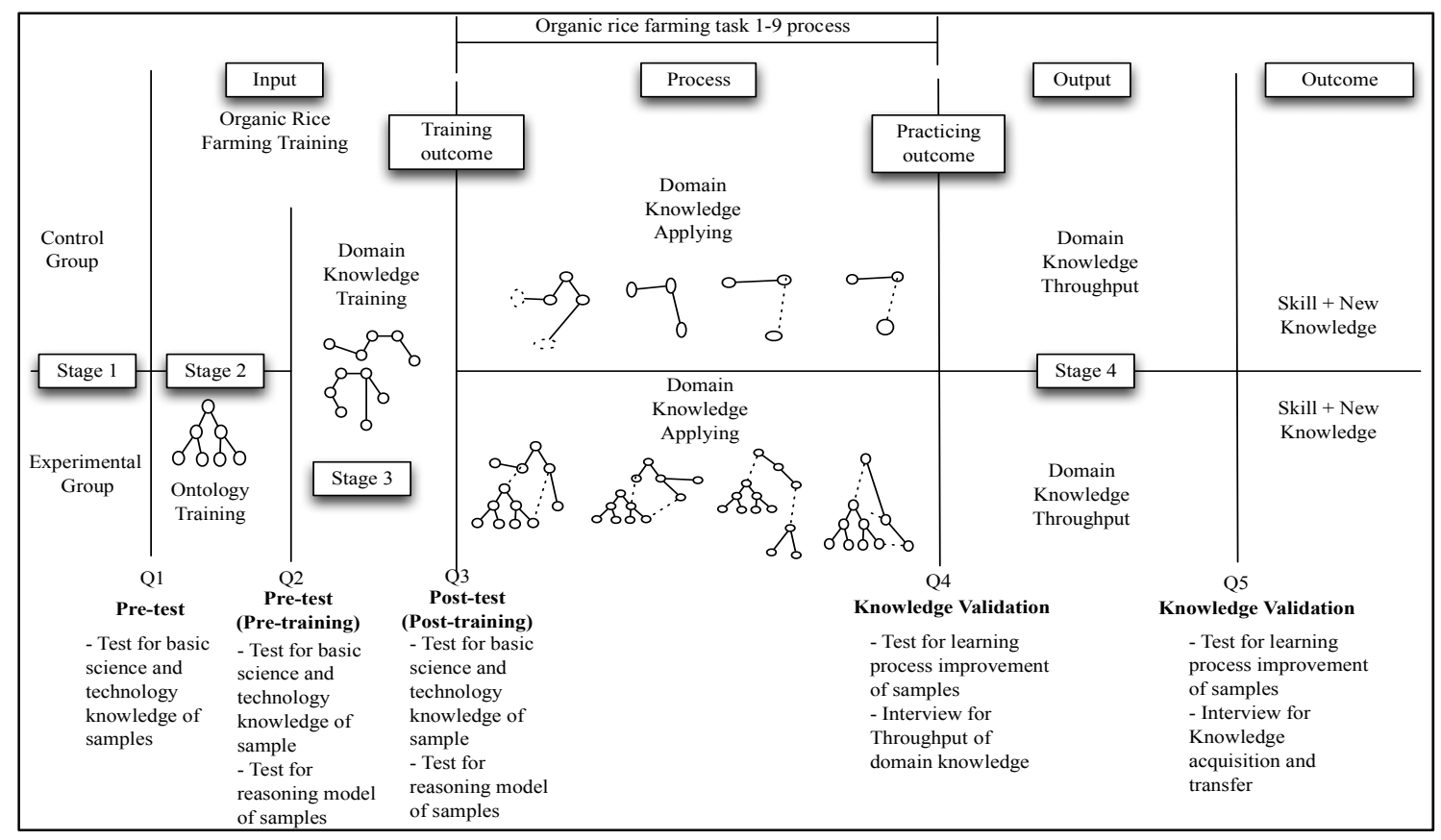

Figure 1. The research methodology and findings verification and validation framework

\subsection{The Sample Qualification: Stage 1}

This research focuses on adaptive organic rice farmers to be a study sample to prove learning skill in learning process from additional ontologies, which was developed. The samples of case study were divided into two groups, which were control group and an experimental group in order to measure the learning process by 
semantic annotation technique on Bloom's Taxonomy. Additionally, this stage involved the reviewing and deciding upon existing knowledge from a sustainable development project which was an organic rice farming knowledge as an appropriate technology.

There are only 37 organic rice farmers in Phrao District community that including farmers in an adaptive step of organic rice farming that are 12 farmers in this organic rice farming community. To qualify sample selection in this research using a test that is designed the questions in science and technology ontology knowledge as following:

1) Which it decompose a fossil in the soil?

2) Which is it decomposed into humus?

3) What are appropriate compositions in soil in agriculture and useful for growing?

4) What are the objectives of cover crop?

5) How to eliminate pets and insects during crop by biological methods?

6) What is microorganism in soil and how microorganism is useful in agriculture?

All 37 organic rice farmers answer 6 questions which these farmers have to response these questions together at the same time in front of the researcher. This research focuses on the answer from 12 adaptive organic rice farmers who can be the research problem representative samples. The qualification of adaptive organic rice farmers must be non-science and technology education or lack of science and technology basic education. The research adaptive organic rice farming samples who could not answer the social science questions will be selected for research examination samples. Then, the adaptive organic rice farmer samples will be divided equally into control and experimental groups.

\subsection{Additional Ontologies for Learners: Stage 2}

The Q1 test provides for both sample groups as a pre-test in order to assess basic understanding of organic rice farming domain knowledge of samples. Then, this stage provides additional ontologies, which were developed for tutoring adaptive organic rice farmer sample in order to prove the hypothesis of ontology effectiveness improving the learning process. Thus, only the experimental group will be tutored 92 additional ontologies of the organic rice farming ontology knowledge based on four main science ontologies derived from the biology, chemistry, physics and mathematics concepts of Thai's curriculum in lower secondary school by a researcher who has science background for one week prior trained domain knowledge. The tutorial of the additional ontology process for samples in an experimental group will take place before the organic rice farming training course from experts domain knowledge both control and experimental groups. The ontology knowledge based on biology, chemistry, physics and mathematics concepts will be train and explain to samples in an experimental group by a researcher who has a Master degree of science. Consequently, the Q2 test will be used to measure basic understanding of organic rice farming domain knowledge of both sample groups to assess the learning process improvement by reasoning domain knowledge with ontology.

\subsection{Organic Rice Agriculture as Appropriate Technology Knowledge Training: Stage 3}

The adaptive organic rice farmer samples in both control and experimental groups were trained together at the same time and the same contents of domain knowledge of intensive organic rice farming tasks by experts who are academic researcher in Chiang Mai University and practitioner in field of appropriate technology knowledge of organic rice farming for one month. The training course and activities are designed to cover all tasks of organic rice farming crop knowledge, which is expected that the learners can understand and apply knowledge of appropriate technology for their organic rice farms.

The term of expert's domain knowledge refers to knowledge which is specific for a given domain of practice, both in terms of more abstract knowledge and domain knowledge embedded in the organizational, social and material context of a given practice. This research has nine tasks of organic rice planting knowledge: T1-soil analysis, T2-seed selection, T3-rice seedling, T4-soil preparation, T5-organic rice growing, T6-water management, T7-rice disease, pet, insect protection, T8-harvest and T9-soil development. Each of 9 tasks was modelled into task, inference, domain knowledge from organic rice farming trainer. Trainers of this research design the training course. Then, the Q3 test will be used as a post-test for training organic rice farming domain knowledge.

\subsection{Learning Process Measurement: Stage 4}

This step provides the measurement of tutorial ontology effectiveness on organic rice farming knowledge in 
learning process improvement of both control and experimental groups. The Q1: pre-test, Q2: Pre-test for pre-training; and Q3: Post-test were designed by experts and used these tests to validate the findings from the experiment. The average throughput of organic rice farming domain knowledge in learning process will be counted and validated in terms of applying domain knowledge, effectiveness domain knowledge with their community and acquiring knowledge by themselves in both control and experimental sample groups. These average throughput of organic rice knowledge at $\mathrm{Q} 4$ to test the learning process improvement.

Additionally, learning process of non-science and technology educated samples will be measured by semantic annotation technique on Bloom's Taxonomy vocabulary. The measurement of learning process of samples by semantic annotation on Bloom's Taxonomy vocabulary: Q4 and Q5 (shown in Figure 2) that it would begin with reading learners' answers from start to end and observing learner's action. The answers and observation of both sample groups would be captured and modelled using CommonKADS then these knowledge models would be manually annotated all annotations in order as they are found to give the most accurate results by semantic annotation measurement on Bloom's Taxonomy vocabulary.

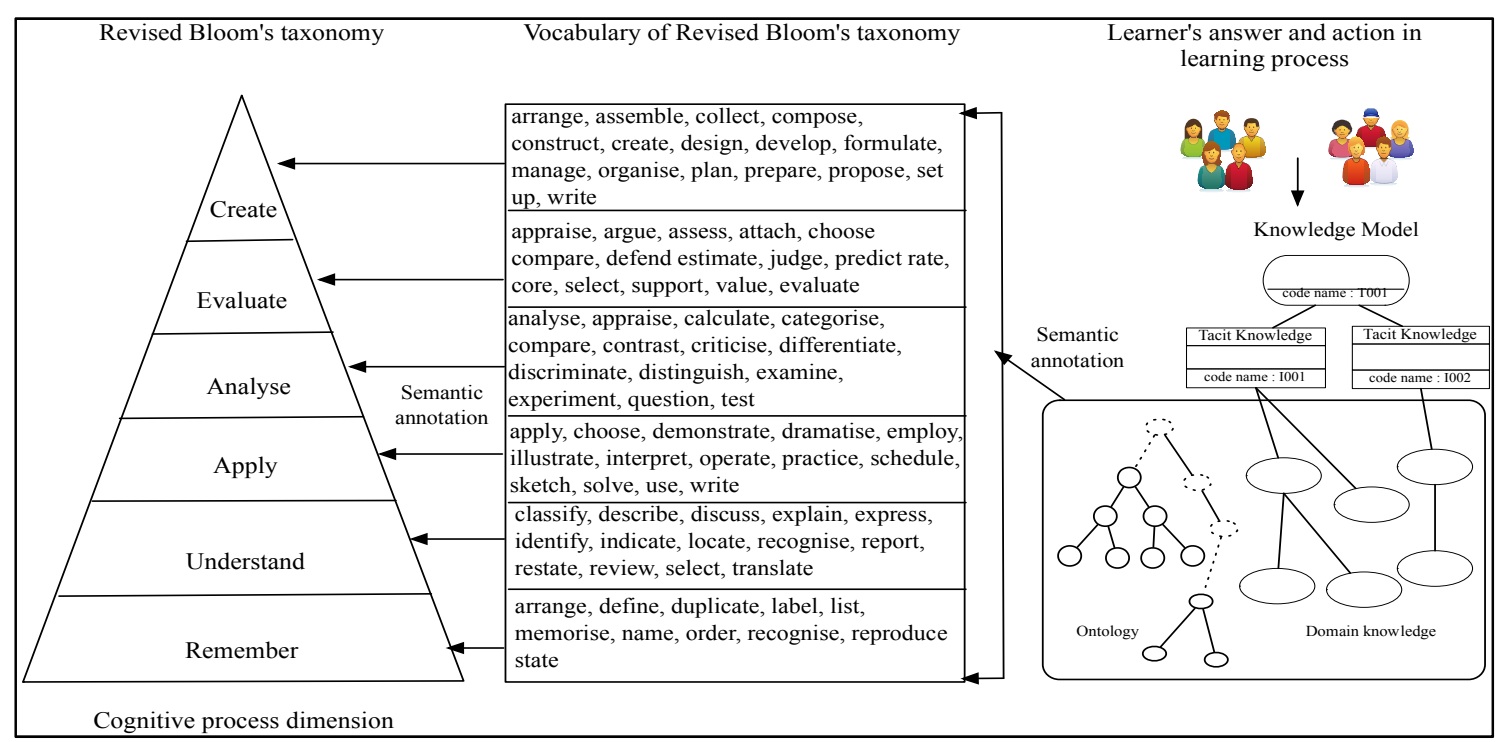

Figure 2. Semantic annotation technique on Bloom's Taxonomy vocabulary

\section{Research Finding}

\subsection{Quality of Samples}

A suitable case study is identified to develop a solution for scientific knowledge. It is Phrao district; Chiang Mai province be selected as an appropriate case study. Phrao District exists partnership and representative of developing an area of Chiang Mai University and use the knowledge from the sustainable development projects to develop the community. Phrao area has a good environment and resources for agriculture that is ready to develop for sustainable living. There are 12,120 families that are 36,393 populations in Phrao District.

There $53.43 \%$ of rural people in Phrao District had the highest education at elementary school level which is a lack of science and technology knowledge. It means that most people in this rural are having the basic education that is not enough to transfer knowledge from appropriate technology successfully for rural development to be a sustainable community. Non-science and technology knowledge educated people do not understand and apply knowledge, which have many conceptualization and social science vocabulary. Consequently, the rural people in Phrao district can be research problem representation because most rural people graduated from primary school and lack of science and technology knowledge. It is envisaged that Phrao District, Chiang Mai Province, Thailand was selected as a case study to transfer organic rice farming knowledge. The knowledge requirement to renew the case study community will be validated by non-structured interviews with stakeholders from the case study.

This study considers an adaptive organic rice-farming group as a learning process technique study sample test because this group characterizes a research problem. This research focused on the answer from 12 adaptive 
organic rice farmers who can be the research problem representative samples. The qualification of adaptive organic rice farmers must be non-science and technology education or lack of science and technology basic education. The 12 adaptive organic rice-farming samples were selected for research examination samples because they could not answer of 6 the social science questions. The test scores of 12 farmers were less than half of full scores that mean they not know and understand about the concept of organic rice farming and lack of science and technology knowledge. There were only 10 adaptive organic rice farmers willing to participate in this research as a research sample. Then, the adaptive organic rice farmer samples were divided equally into control and experimental groups.

\subsection{Learning Process Measurement}

\section{Effectiveness of tutorial social science ontology in learning process}

The learning process was designed to measure the ontology effectiveness and the sample learning method that following only the experts or adopting the domain knowledge by using ontology in reasoning. The only the experimental group was tutored the social science ontologies of organic rice farming to prove the ontology effectiveness. Then, the control and experimental groups were trained in organic rice farming by experts and were tested for knowledge gained from trainers following Bloom's Taxonomy framework. There was 148-domain knowledge of nine intensive tasks of organic rice farming which were disseminated to both sample groups. The experimental group only was trained 92 ontologies based on science concepts on organic rice farming. The training outcome of the learning process was assessed via capturing organic rice domain knowledge from both sample groups using CommonKADS.

The domain knowledge reasoning with ontology mapping in each test of Q1-Q5 is a set of expressions that has a different ontology of both sample groups, which were mapped in learning process in every task of organic rice agriculture tasks. The organic rice farming domain knowledge of both adaptive farmer sample groups were elicited and captured via interview using CommonKADS to map learning outcome defining with jargon of experts using manual semantic annotation to get effectiveness domain knowledge and comparing between one by one of each control group and experimental group. The example of the comparison of domain knowledge reasoning with ontology of one pair sample from both groups that is shown in Figure 3).

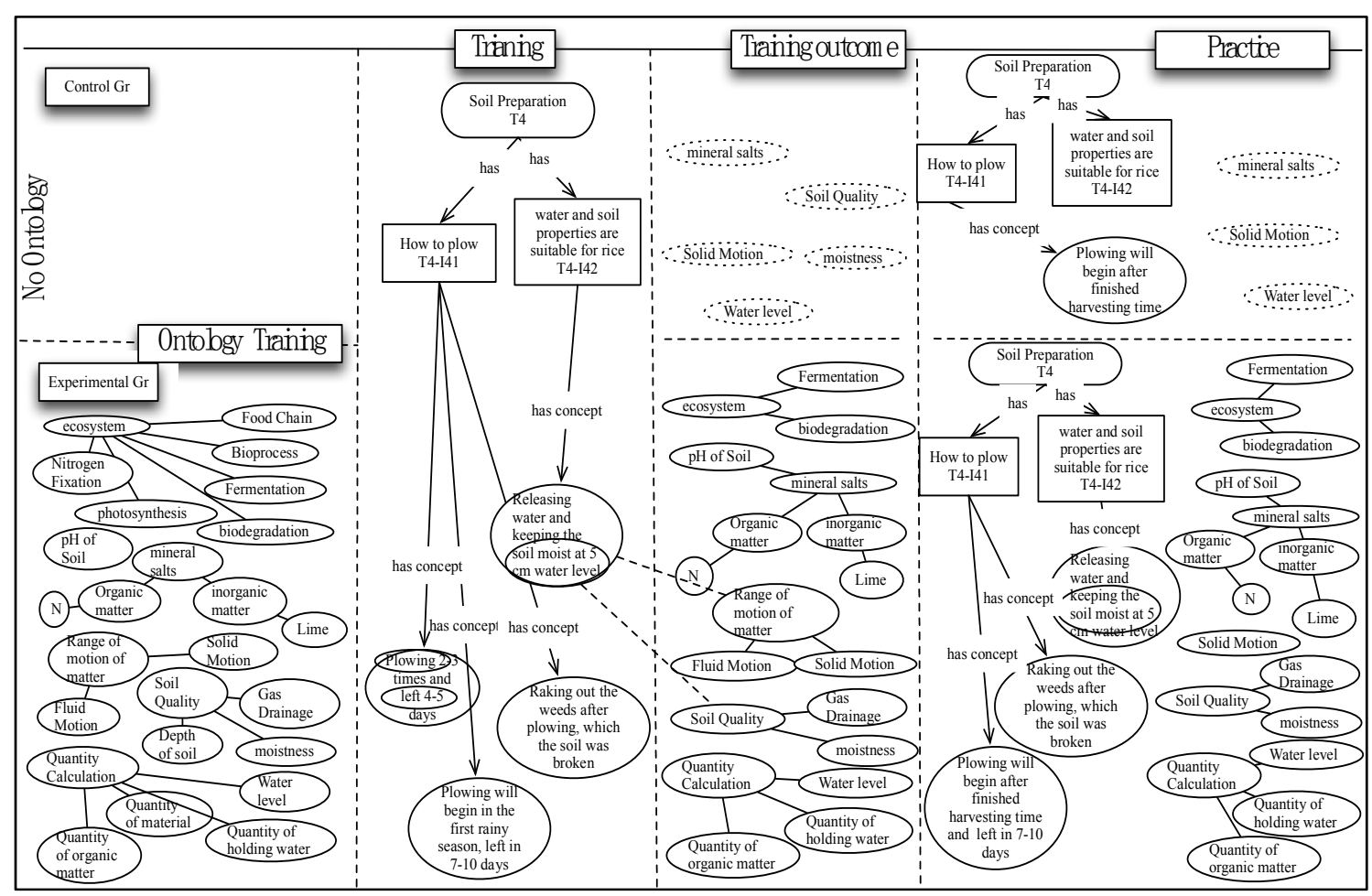

Figure 3. The map of domain knowledge validation of organic rice farming knowledge from both control and experimental groups 
The findings were verified by experts who have experience in appropriate technology knowledge of every step of this research. The research findings were validated using test, pre-test and post-test that was design by experts. The scores of all tests in each sample group are showed in Table 1. The results of knowledge validation with pre-test and post-test average scores showed that experimental sample group that had tutorial science ontologies has better scores more than control group. These findings mean the experimental group that can be reasoning domain knowledge with science ontologies and show the effectiveness of tutorial science ontologies. The results of social science ontology effectiveness in learning process of test Q1, Q2 and Q3 showed that the experimental group which is with ontology training can remember, understand and practice knowledge from experts more than the control group which is without tutorial social science ontologies (shown in Table 1). The average number of organic rice farming domain knowledge in learning process was counted and validated with tests in terms of domain knowledge in both control and experimental groups. The both sample groups took the test Q1 as a pre-test, Q2 as pre-training (experimental group has already been trained ontologies), then Q3 as post-test after training. Consequently, all answers would be captured using knowledge model of CommonKADS into task, inference and domain knowledge and then using manual semantic annotation technique to annotate domain knowledge from sample's answer with experts' jargons to assess ontology reasoning of sample.

Table 1. The test scores of control and experimental group in each stage

\begin{tabular}{lcccc}
\hline & $\begin{array}{c}\text { Mean of control } \\
\text { group }\end{array}$ & $\begin{array}{c}\text { \% of Mean of control } \\
\text { group }\end{array}$ & $\begin{array}{c}\text { Mean of experimental } \\
\text { group }\end{array}$ & $\begin{array}{c}\text { \% of Mean of experimental } \\
\text { group }\end{array}$ \\
\hline The scores of test (Q1) & 47 & 31.48 & 48 & 32.22 \\
\hline $\begin{array}{l}\text { The scores of Pre-test } \\
\text { (Q2) }\end{array}$ & 47 & 31.91 & 69 & 46.81 \\
\hline $\begin{array}{l}\text { The scores of Post-test } \\
\text { (Q3) }\end{array}$ & 63 & 42.47 & 91 & 61.49 \\
\hline
\end{tabular}

The Wilcoxon Signed Ranks statistic approach was used to compare the mean difference on number of domain knowledge in Q1, Q2 and Q3 between the control and experimental group that tests to prove the tutorial ontology effectiveness hypothesis. The significance of the two-sided test shows in Table 2 that the median of the differences between Q1 and Q2 of both sample groups is 0.025 at $\alpha=0.05$ and the median of the differences between Q2 and Q3 of both sample groups is 0.005 at $\alpha=0.05$, so it can be seen that the mean difference on average domain knowledge between experimental group and control group is totally different and statistically significant. In addition, this statistical approach shows that the tutorial social science ontology effectiveness as a study hypothesis has been proved via the throughput domain knowledge counting in learning process to be statically significant of domain knowledge.

Table 2. Statistical test of the domain knowledge measurement between control and experimental group comparison of Q1, Q2 and Q3 test

\begin{tabular}{lll}
\hline & Pre-test Q1-Post-test Q2 & Pre-test Q2-Post-test Q3 \\
\hline Exact Sig. [2*(1-tailed Sig.) $]$ & .025 & .005 \\
\hline
\end{tabular}

The Q4 and Q5 were used to validate learning process output and outcome. The average throughput of organic rice farming domain knowledge in learning process was counted and validated in terms of applying domain knowledge, effectiveness domain knowledge with their community and acquiring knowledge by themselves in both control and experimental sample groups. The results of ontology effectiveness in learning process showed that the experimental group which was with social science ontologies training could understand and apply knowledge from experts more than the control group which was without tutorial ontologies (shown in Table 3). The experimental group only had tutorial ontology on organic rice farming as it can be seen in mapping in Figure 3. The adaptive farmer samples in this group could remember and reason ontologies to understand expert's concept and create their own domain knowledge, which was appropriate to their community. 
Table 3. Throughput of organic rice farming domain knowledge comparison between control and experimental groups (Q4 and Q5)

\begin{tabular}{lcccc}
\hline & $\begin{array}{c}\text { Mean of control } \\
\text { group }\end{array}$ & $\begin{array}{c}\text { Mean of experimental } \\
\text { group }\end{array}$ & $\begin{array}{c}\text { Percentage of control } \\
\text { group }\end{array}$ & $\begin{array}{c}\text { Percentage of experimental } \\
\text { group }\end{array}$ \\
\hline $\begin{array}{l}\text { Number of applying domain } \\
\text { knowledge (Q4) }\end{array}$ & 19.2 & 53.40 & 12.97 & 36.08 \\
\hline $\begin{array}{l}\text { Number of effective domain } \\
\text { knowledge (Q4) }\end{array}$ & 11 & 26.20 & 11.00 & 17.70 \\
\hline $\begin{array}{l}\text { Number of acquiring domain } \\
\text { knowledge (Q5) }\end{array}$ & 0 & 8.20 & 0 & 5.54 \\
\hline
\end{tabular}

The additional ontologies on organic rice farming knowledge was validated via a count of number of using domain knowledge using CommonKADS to annotate domain knowledge from both control and experimental groups with experts' jargons (shown in Figure 3). Some ontology could not be used on organic rice appropriately, and some ontology of their adaptive organic rice farmer samples could be reasoned and created which was related to expert's domain knowledge to apply for organic rice farming. Furthermore, the new domain knowledge on organic rice farming which is suitable for case study was created from non-science and technology educated farmers in experimental sample group. The results of ontology effectiveness in learning process show that the experimental group which was with ontology training could understand and apply knowledge from trainers more than the control group effectively which was without tutorial ontologies (shown in Table 1 and 3). The average throughput of organic rice farming domain knowledge in learning process was counted and validated in terms of applying domain knowledge (Q4), effective domain knowledge (Q4) with their community and acquiring knowledge (Q5) by themselves in control group and experimental group. The total number domain knowledge from experts was 148 domain knowledge to train in learning process for both control and experimental sample groups, then the output of average of organic rice farming domain knowledge of learning process learned by both sample groups was counted as number of throughput domain knowledge (shown in Figure 4) and calculated as percentage (shown in Figure 5).

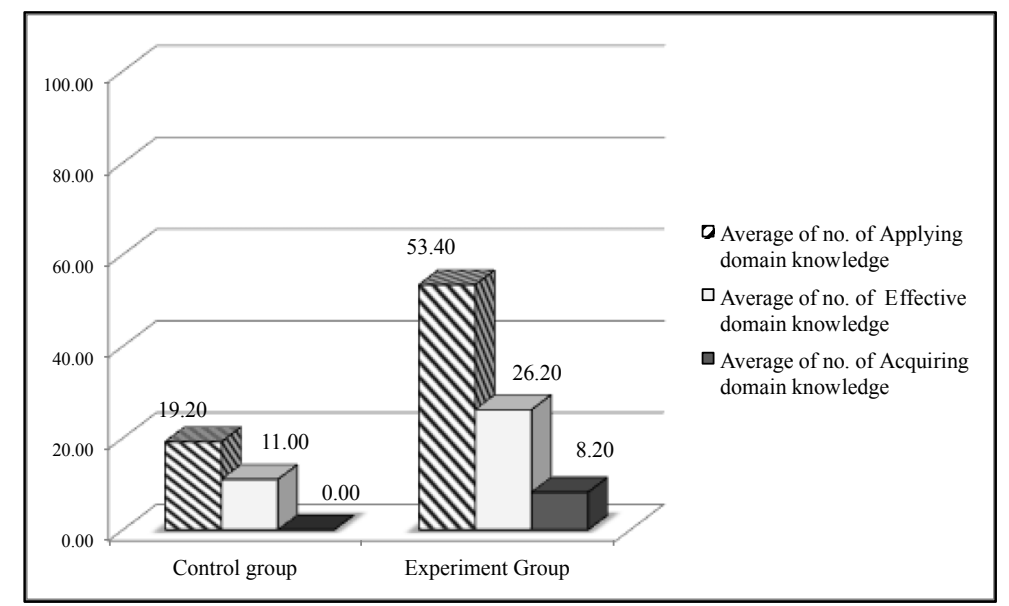

Figure 4. Comparison of domain knowledge on organic rice farming of control and experimental group 


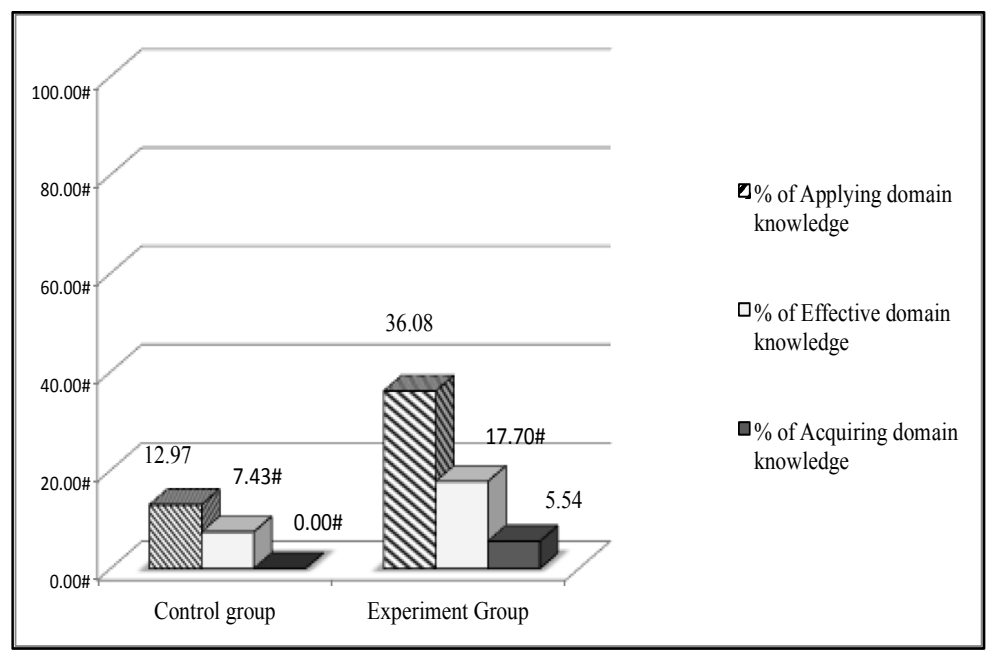

Figure 5. Comparison of domain knowledge percentage on organic rice farming between control and experimental group

Table 4. Statistical test of the throughput domain knowledge measurement between control and experimental group comparison

\begin{tabular}{lccc}
\hline & $\begin{array}{c}\text { Number of applying domain } \\
\text { knowledge }(\mathrm{Q} 4)\end{array}$ & $\begin{array}{c}\text { Number of effective domain } \\
\text { knowledge }(\mathrm{Q} 4)\end{array}$ & $\begin{array}{c}\text { Number of acquiring domain } \\
\text { knowledge (Q5) }\end{array}$ \\
\hline $\begin{array}{l}\text { Exact Sig. }[2 *(1 \text {-tailed } \\
\text { Sig.) }]\end{array}$ & .008 & .008 & .008 \\
\hline
\end{tabular}

The Wilcoxon Signed Ranks statistic approach was used to compare the mean difference on number of applying, effective and acquiring domain knowledge between the control and experimental group that tests to prove the tutorial ontology effectiveness hypothesis. The significance of the two-sided test shows in Table 4 that the median of the differences is 0.008 at $\alpha=0.05$, so it can be seen that the mean difference on average throughput domain knowledge between experimental group and control group is totally different and statistically significant. In addition, this statistical approach shows that the tutorial social science ontology effectiveness as a study hypothesis has been proved via the throughput domain knowledge counting in learning process to be statically significant of applying, effective and acquiring domain knowledge which in applying cognitive level on Bloom's Taxonomy.

\section{Semantic annotation technique on Bloom's Taxonomy vocabulary}

The measurement of learning process improvement of both non-science and technology educated control and experimental sample groups by semantic annotation on Bloom's Taxonomy vocabulary (shown in Figure 6) suggested that it would begin with reading learners' answers researcher's question and interview agendas which follows Bloom's Taxonomy vocabulary from start to end and observing learner's actions. The answers and observation of both sample groups were captured and modelled using CommonKADS, then these knowledge models were manually semantic annotated all annotations as they are found to give the most accurate results by semantic annotation measurement on Bloom's Taxonomy vocabulary. 


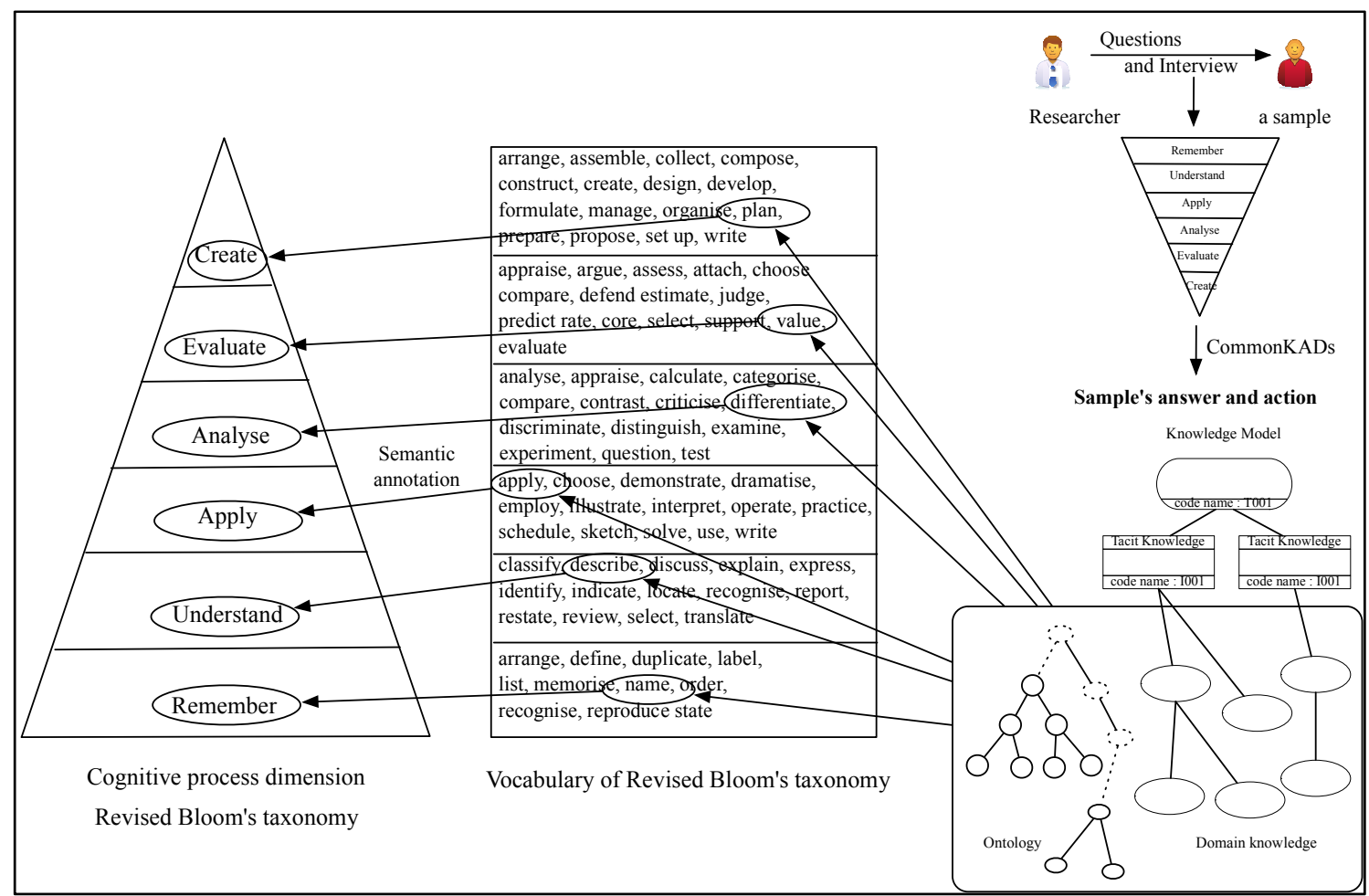

Figure 6. The mapping of semantic annotation technique on Bloom's Taxonomy vocabulary to cognitive level

The questions were designed using the behaviour vocabulary of Bloom's Taxonomy, then tested for both control and experimental groups. The cognitive level of each adaptive organic rice farmer sample was defined from the answers and actions by semantic annotation on revised Bloom's Taxonomy following Table 5 such as if a sample was questioned "Can you describe how to develop organic rice farm soil?" and a sample can describe obviously of organic rice soil development that means the sample is in understanding level of Bloom's Taxonomy. The ten samples of both non-science and technology educated organic rice sample groups were assessed by semantic annotation on revised Bloom's Taxonomy vocabulary in order to define the cognitive level of each sample in learning process. The result shows in Figure 8 that all five samples in experimental group were in analysis cognitive level and just three samples could reach to creating cognitive level. The three samples in control group were in remember cognitive level and no samples in this control group could reach to analysis, evaluating and creating cognitive level.

Table 5. The revised Bloom's Taxonomy

\begin{tabular}{ccc}
\hline $\begin{array}{c}\text { Cognitive } \\
\text { level }\end{array}$ & Description & Learner's answer and action \\
\hline
\end{tabular}

Remember

Ability to recognise, arrange trained material, memorize of definitions that without an understanding of the related meaning.

Understand

Ability to understand, explain and indicate the trained material.

Apply
Ability to practise the trained material to apply in new situations.
Recall the ontology in the exact content that it was trained on organic rice farming.

Restate the trained material in the learner's own vocabulary or can discuss unseen concept of organic rice farming.

Apply the appropriate technology of trained material to operate the learner own organic rice farm. 
Ability to distinguish and examine

Analyse complex concepts or situations into the learner component portions.

Ability to judge and evaluate the worth

Evaluate of trained concepts, knowledge, etc. for specified determination.

Ability to propose or rearrange

Create component portions to create new concepts.
Compare or contrast the performance of the trained organic rice farming to learner component portions and analyse the related component parts to their own situations or another.

Evaluate the business outcome of the learner own organic rice products and making judgments on the quality of their own products.

Create and develop their own organic rice farming domain knowledge based on appropriate technology concepts.

Reference: Bloom et al. (1956), Anderson and Krathwohl (2001).

The example of the learning process measurement by innovative semantic annotation on Bloom's Taxonomy vocabulary is shown in Figure 7 and the evaluation form of cognitive level was shown in Table 6. The ten samples of both non-science and technology educated organic rice sample groups were assessed by semantic annotation on revised Bloom's Taxonomy in order to define the cognitive level of each sample in learning process.

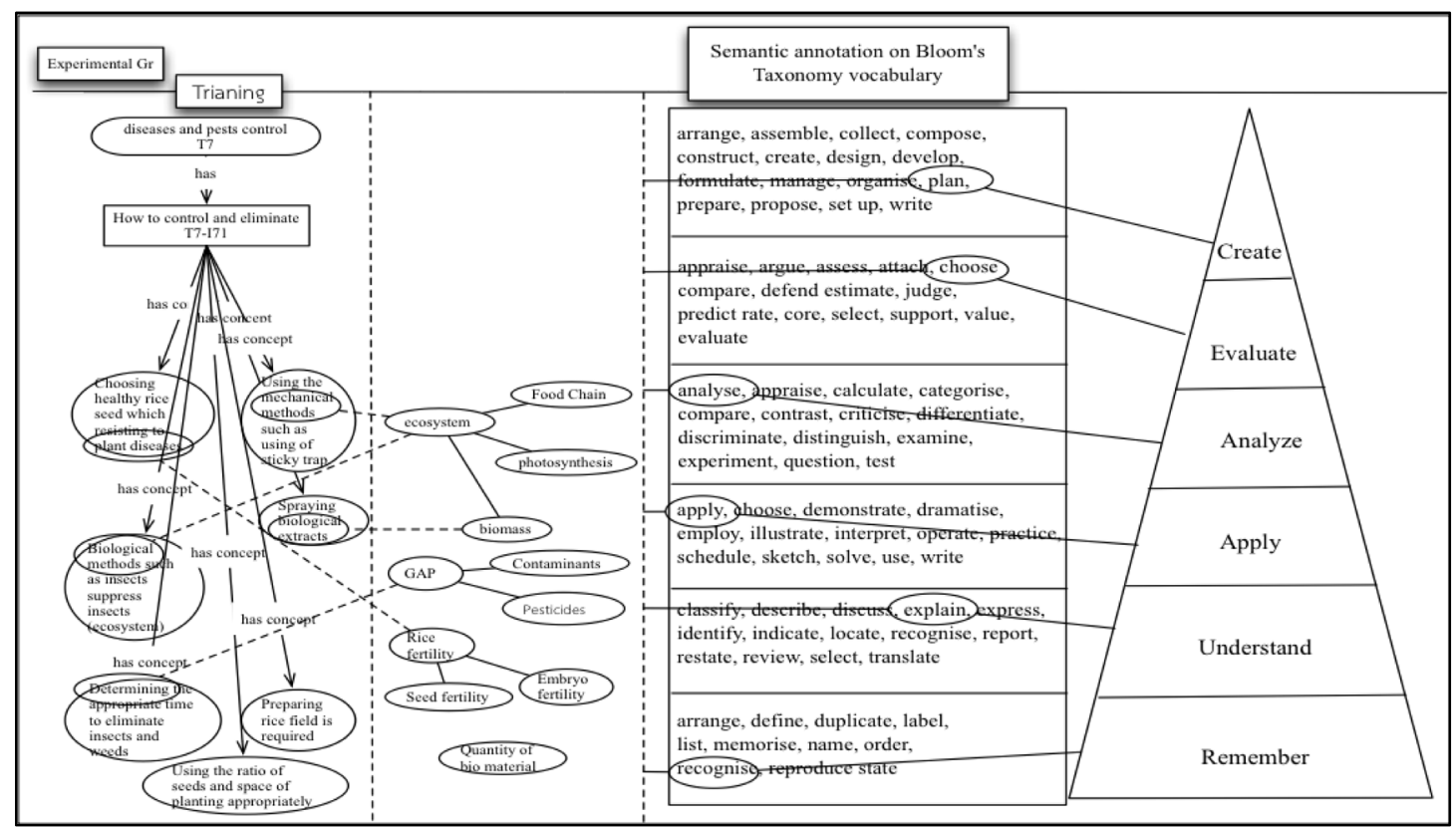

Figure 7. The example of mapping of semantic annotation technique on Bloom's Taxonomy vocabulary to cognitive level of the sample in experimental group who has tutorial ontologies on task 7 diseases and pets' control 
Table 6. A cognitive behaviour evaluation for non-science and technology educated farmers of organic rice farming learning process improvement

\begin{tabular}{|c|c|c|c|c|}
\hline $\begin{array}{l}\text { Cognitive } \\
\text { behaviour } \\
\text { indicator of } \\
\text { Bloom's } \\
\text { Taxonomy }\end{array}$ & $\begin{array}{l}\text { Fail (0 } \\
\text { points) }\end{array}$ & Fair (1 points) & Pass ( 2 points $)$ & Good (3 points) \\
\hline Remember & & & & \\
\hline $\begin{array}{c}\text { Understand } \\
\text { Apply } \\
\text { Analyze } \\
\text { Evaluate } \\
\text { Create }\end{array}$ & $\begin{array}{l}\text { Could not } \\
\text { answer all } \\
\text { questions }\end{array}$ & $\begin{array}{l}\text { Answers are not clearly } \\
\text { and get to the key words } \\
\text { of correct answers, } \\
\text { Confusing answers }\end{array}$ & $\begin{array}{l}\text { Answers get to the } \\
\text { points of questions } \\
\text { but it is still needed } \\
\quad \text { more detail }\end{array}$ & $\begin{array}{c}\text { Answers are completely } \\
\text { and covered for all } \\
\text { aspect of questions, } \\
\text { Clearly answers }\end{array}$ \\
\hline
\end{tabular}

All answers from both sample groups were captured using CommonKADS into task, inference and domain and then using the semantic annotation technique on Bloom's Taxonomy vocabulary to measure and score learning process improvement of all samples. The experts and researcher assessed the learning process improvement of all samples using the evaluation form in Table 6 to score the clearly fine answer together with semantic annotation on Bloom's Taxonomy vocabulary. For example, in the remember cognitive level on Bloom's Taxonomy, the sample can recognize the all domain knowledge of organic rice farming and the answers are complete, covered for all tasks of organic rice farming knowledge and clearly answers then the sample should be scored at 3 points in good level. If the sample cannot recognize and could not answer all questions, which based on Bloom's Taxonomy vocabulary, the sample's score would be zero point. Every cognitive level of all samples on Bloom's Taxonomy was evaluated by semantic annotation on Bloom's Taxonomy vocabulary together with cognitive behaviour evaluation.

The total evaluation scores of all organic rice farming knowledge in task 1 to task 9 were calculated from learning process measurement by semantic annotation on Bloom's Taxonomy vocabulary using assessment form of both sample groups using assessment form of both sample groups shown in Table 7 .

Table 7. Total evaluation scores of all organic rice farming tasks (Task 1-Task 9) were calculated from learning process measurement by semantic annotation on Bloom's Taxonomy vocabulary using assessment form of both sample groups using assessment form of both sample groups

\begin{tabular}{lllllllllll}
\hline \multirow{2}{*}{ Cognitive Level } & \multicolumn{4}{c}{ Control Group (points) } & \multicolumn{5}{c}{ Experimental Group (points) } \\
\cline { 2 - 10 } & AD1 & AD2 & AD3 & AD4 & AD5 & AD6 & AD7 & AD8 & AD9 & AD10 \\
\hline Remember & 6 & 3 & 0 & 1 & 0 & 14 & 15 & 13 & 15 & 12 \\
Understand & 2 & 1 & 0 & 1 & 0 & 10 & 12 & 9 & 9 & 9 \\
Apply & 2 & 0 & 0 & 1 & 0 & 7 & 7 & 5 & 7 & 5 \\
Analyze & 0 & 0 & 0 & 0 & 0 & 2 & 4 & 2 & 4 & 2 \\
Evaluate & 0 & 0 & 0 & 0 & 0 & 2 & 1 & 0 & 1 & 1 \\
Create & 0 & 0 & 0 & 0 & 0 & 2 & 2 & 0 & 1 & 0 \\
\hline
\end{tabular}

From Table 7, it can be seen that the learning process of samples in an experimental group having additional ontology are improvement more than control group significantly. The semantic annotation technique on Bloom's Taxonomy vocabulary can measure the learning process improvement and prove the ontology effectiveness. The number of sample in control and experimental groups who can improve their learning process by reasoning domain knowledge with ontology and reach the cognitive level on Bloom's Taxonomy shows in Table 8. 
Table 8. Number of sample in each group reaches the cognitive level

\begin{tabular}{lccc}
\hline Cognitive Level & Number of sample in control group (person) & $\begin{array}{c}\text { Number of sample experimental group } \\
\text { (person) }\end{array}$ \\
\hline Remember & 3 & 5 \\
Understand & 2 & 5 \\
Apply & 2 & 5 & 5 \\
Analyze & 0 & & 4 \\
Evaluate & 0 & 3 & \\
Create & 0 & & \\
\hline
\end{tabular}

The result in Table 8 and Figure 8 showed that all five samples in experimental group were in analysis cognitive level and just three samples could reach to creating cognitive level. The three samples in control group were in remember cognitive level and no samples in this control group could reach to analysis, evaluating and creating cognitive level.

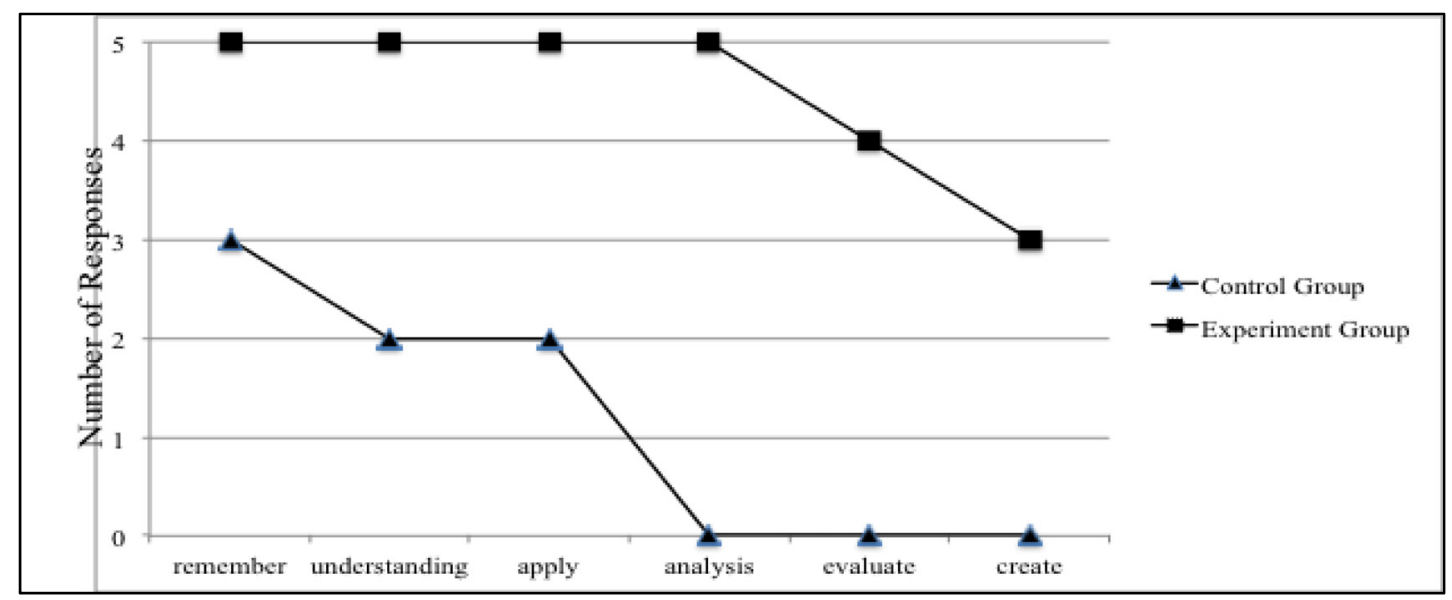

Figure 8. Comparison of cognitive level of both control and experimental groups

\section{Discussion and Conclusion}

This study has mainly concerned with measurement of effective additional ontology which a commonly-agreed understanding of expert's jargons (domain knowledge) that can be shared, reasoned, reused and operationalized across communities in learning process by semantic annotation technique on Bloom's Taxonomy vocabulary using CommonKADS in order to assess learning process improvement of samples.

The test at Q2, Q3, Q4 and Q5 scores of learning process measurement by semantic annotation on Bloom's Taxonomy vocabulary showed that additional ontologies could improve learning process behaviour of samples as sample in experimental group. The measurement of learning process of both non-science and technology educated control and experimental sample groups used semantic annotation on Bloom's Taxonomy vocabulary as research innovative assessment to identify the cognitive level of each sample in both groups in learning process improvement. The answers and observation of all adaptive organic rice farmer samples were modelled using CommonKADS, then these domain knowledge models were manually semantic annotated with Bloom's Taxonomy vocabulary together with scored evaluation. The all five adaptive farmer samples in an experimental group that were tutored additional ontologies are in analysis cognitive level and just three samples could extend to creating cognitive level of Bloom's Taxonomy. There were no samples in control group, which could reach to analysis, evaluating and creating cognitive level on Bloom's Taxonomy.

The average throughput of organic rice farming domain knowledge in learning process provided applying cognitive level of Bloom's Taxonomy was counted and validated in terms of applying domain knowledge (Q4), effective domain knowledge (Q4) with their community and acquiring knowledge (Q5) by themselves in control 
group and experimental group. The tutorial social science ontology effectiveness on organic rice farming knowledge was validated via a count of number of applying domain knowledge by semantic annotation using CommonKADS from both control and experimental groups effectively to measure the ontology effectiveness in learning process. The research results of ontology effectiveness in learning process improvement showed that the experimental group that was with ontology training could understand and apply knowledge from trainers better than the control group, which was without tutorial social science ontologies. This study focused on the benefits of ontologies in supporting knowledge for non-science and technology educated farmers. The tutorial ontology effectiveness and cognitive level of samples were assessed using the measurement in learning process by semantic annotation technique on Bloom's Taxonomy vocabulary. The results defined in the ontologies effectiveness which improved learning process, supported and enhanced understanding the organic rice farmers in Phrao District, Chiang Mai Province as knowledge workers could also use the additional ontologies as a vocational learning tool to improve their competency and distribute domain knowledge to people in order to develop and solve the problem issue in their community. Non-science and technology educated farmers can learn from this domain knowledge structure by reasoning ontology to improve and develop their communities which are measured by semantic annotation technique on Bloom's Taxonomy vocabulary using a CommonKADS as innovative of this research which it provides tools to support structuring knowledge and identify cognitive level of learners in learning process. The measurement of learning process by semantic annotation on Bloom's Taxonomy vocabulary can prove that the additional ontologies could improve the learning process of non-science and technology educated farmers.

\section{References}

Airasian, P. W., \& Miranda, H. (2002). The Role of Assessment in the Revised Bloom's Taxonomy. Theory into Practice, 41(4), 349-354. http://dx.doi.org/10.1207/s15430421tip4104_8

Anderson, L. W., Krathwohl, D. R., Airasian, P. W., Cruikshank, K. A., Mayer, R. E., Pintrich, P. R. et al. (2001). A taxonomy for learning, teaching and assessing: A revision of Bloom's taxonomy of educational objectives. New York: Longman.

Anderson, W., \& Krathwohl, D. R. (2008). A taxonomy for learning, teaching, and Assessing: A revision of Blooms'Educational Objectives. New York, NY: Longman, 2001CSIR Built Environment Unit, Appropriate Technologies in the Water Sector in South Africa, May.

Angele, J., Fensel, D., Landes, D., \& Studer, R. (1998). Developing knowledge based systems with MIKE. Journal of Automated Software Engineering, 5(4), 389-418. http://dx.doi.org/10.1023/A:1008653328901

Anumba, C. J., Issa, R. A., Pan, J., \& Mutis, I. (2008). Ontology-based information and knowledge management in construction. International Journal of Construction Innovation, 8(3), 218-239. http://dx.doi.org/10.1108/14714170810888976

Bloom, B. S., Engelhart, M. D., Furst, E. J., Hill, W. H., \& Krathwohl, D. R. (1956). Taxonomy of educational objective: The classification of educational goals. New York: Longman.

Box, I. (2004). Objective-Oriented Analysis, Criterion Referencing, and Bloom. Proceedings of the sixth conference on Australian computing education, 30, 1-8.

Chou, T. H., Vassarand, J. A., \& Lin, B. (2008). Knowledge management via ontology development in $\begin{array}{lllll}\text { accounting. International Journal of } & \text { Kybernetes, } & 37(1), & 36-48 .\end{array}$ http://dx.doi.org/10.1108/03684920810850970

Chyung, S. Y., \& Stepich, D. (2003). Applying the “Congruence” Principle of Bloom's Taxonomy to Designing Online Instruction. The Quarterly of Distance Education, 4(3), 317-330.

Gennari, J. H., Musen, M. A., Fergerson, R. W., Grosso, W. E., Crubezy, M., Eriksson, H., . . Tu, S. W. (2003). The evolution of protege: An environment for knowledge-based systems development. International Journal of Human Computer, 58(1), 89-123. http://dx.doi.org/10.1016/S1071-5819(02)00127-1

Gruber, T. R. (1993). Towards principles for the design of ontologies used for knowledge sharing. In N. Guarino \& R. Poli (Eds.), Formal Ontology in Conceptual Analysis and Knowledge Representation. Kluwer Academic Publishers.

Handschuh, S. (2005). Creating Ontology-based Metadata by Annotation for the Semantic Web (Ph.D. thesis, University of Karlsruhe).

Krathwohl, D. R. (2002). A revision of Bloom's taxonomy: An overview. Theory into Practice, 41, 212-218. http://dx.doi.org/10.1207/s15430421tip4104_2 
Kunen, S., Cohen, R., \& Solman, R. (1981). A levels-of-processing analysis of Bloom's Taxonomy. Journal of Educational Psychology, 73, 202211. http://dx.doi.org/10.1037/0022-0663.73.2.202

Lister, R., \& Leaney, J. (2003). Introductory Programming, Criterion-Referencing, and Bloom. Proceedings of SIGCSE 2003, ACM Press (pp. 143-147). http://dx.doi.org/10.1145/611892.611954

Liyabage, L., Strachan, R., Penlington, R., \& Casselden, B. (2013). Design of Educational Systems for Work Based Learning (WBL): the Learner Experience. High Education, Skills and Work-Based Learning, 3(1), 51-61. http://dx.doi.org/10.1108/2042389 1311294984

Martine, C. (1999). Methodology and tools oriented to knowledge engineering applications. MOKA public report No.2 [online]. Retrieved from http://www.kbe.conventry.ac.uk/MOKA

Mayer, R. E. (2002). A Taxonomy for Computer-Based Assessment of Problem Solving. Computers in Human Behavior, 18(6), 623-632. http://dx.doi.org/10.1016/S0747-5632(02)00020-1

Pappas E., Pierrakos, O., \& Nagel R. (2013). Using Bloom's Taxonomy to Teach Sustainability in Multiple Contexts. Journal of Cleaner Production, 48, 54-64. http://dx.doi.org/10.1016/j.jclepro.2012.09.039

Plack, M. M., Driscoll, M., Marquez, M., Cuppernull, L., Maring, J., \& Greenberg, L. (2007). Assessing Reflective Writing on a Pediatric Clerkship by Using a Modified Bloom's Taxonomy. Ambulatory Pediatrics, 7(4), 285-291. http://dx.doi.org/10.1016/j.ambp.2007.04.006

Saito A., Umemoto, K., \& Ikeda, M. (2007). A strategy-based ontology of knowledge management technologies. Journal of Knowledge Management, 11(1), 97-114. http://dx.doi.org/ 10.1108/13673270710728268

Schreiber, A. Th., Akkermans, J., Anjewierden, A., de Hoog, R., Shadbolt, N., van de Velde, W., \& Wielinga, B. (2000). Knowledge engineering and management: The CommonKADS methodology. MIT Press.

Scott, T. (2003). Bloom's Taxonomy Applied to Testing in Computer Classes. The Journal of Computing in Small Colleges, 19(1), 267-274.

Uren, V., Cimiano, P., Iria, J., Handschun, S., Vargas-Vera, M., Motta, E., \& Ciravegna, F. (2006). Semantic annotation for knowledge management: requirements and a survey of the state of art. Journal of web semantics, 4, 14-28. http://dx.doi.org/ 10.1016/j.websem.2005.10.002

Uschold, M., \& Gruninger, M. (1996). Ontologies: Principles, Methods and Applications. Knowledge Engineering Review, 11(2), 93-115. http://dx.doi.org/10.1017/S0269888900007797

Valcke, M., De Wever, B., Zhu, C., \& Deed, C. (2009). Supporting Active Cognitive Processing in Collaborative Groups: The Potential Bloom's Taxonomy as a Labeling Tool. Internet and Higher Education, 12, 165-172. http://dx.doi.org/10.1016/j.iheduc. 2009.08.003

\section{Copyrights}

Copyright for this article is retained by the author(s), with first publication rights granted to the journal.

This is an open-access article distributed under the terms and conditions of the Creative Commons Attribution license (http://creativecommons.org/licenses/by/3.0/). 